\title{
Low energy dipole strength from large scale shell model calculations
}

\author{
Kamila Sieja ${ }^{\mathrm{a}}$ \\ Université de Strasbourg, IPHC, 23 rue du Loess CNRS, UMR7178, 67037 Strasbourg, France
}

\begin{abstract}
Low energy enhancement of radiative strength functions has been deduced from experiments in several mass regions of nuclei. Such an enhancement is believed to impact the calculated neutron capture rates which are crucial input for reaction rates of astrophysical interest. Recently, shell model calculations have been performed to explain the upbend of the $\gamma$-strength as due to the $M 1$ transitions between closelying states in the quasi-continuum in Fe and Mo nuclei. Beyond mean-field calculations in Mo suggested, however, a non-negligible role of electric dipole in the low energy enhancement. So far, no calculations of both dipole components within the same theoretical framework have been presented in this context. In this work we present newly developed large scale shell model appraoch that allows to treat on the same footing natural and non-natural parity states. The calculations are performed in a large $s d-p f-g d s$ model space, allowing for $1 \mathrm{p}-1 \mathrm{~h}$ excitations on the top of the full $p f$-shell configuration mixing. We restrict the discussion to the magnetic part of the dipole strength, however, we calculate for the first time the magnetic dipole strength between states built of excitations going beyond the classical shell model spaces. Our results corroborate previous findings for the $M 1$ enhancement for the natural parity states while we observe no enhancement for the $1 \mathrm{p}-1 \mathrm{~h}$ contributions. We also discuss in more detail the effects of configuration mixing limitations on the enhancement coming out from shell model calculations.
\end{abstract}

\section{Introduction}

Photoneutron cross sections and radiative neutron capture cross sections are fundamental nuclear inputs to stellar model calculations of nucleosynthesis processes. Evaluation of (n, $\gamma$ ) cross sections requires a knowledge of statistical nuclear quantities, such as $\gamma$-ray strength function $(\gamma$-SF $)$ and nuclear level density. The $\gamma$-SF below the neutron threshold plays a key role in defining the electromagnetic de-excitation taking place after the neutron capture, which, together with the $\beta$-decay, drives the $s-$ and $r$-process nucleosynthesis.

The nuclear shell model, known as configuration interaction in other fields, is commonly used to calculate nuclear spectra and transitions as well as strength distributions of electromagnetic operators. Recently, a method of obtaining the dipole strength functions from averages of a large number of $M 1$ transitions within the shell model has been proposed [1]. It has been demonstrated for mass $A \sim 90$ region that the $M 1$ $\gamma$-SF are enhanced toward low energy, in agreement with experimental observations. It has been suggested that it happens in nuclei near closed shells where high $j$ proton and neutron orbitals are located near the Fermi surface with magnetic moments adding up coherently. Later calculations in iron nuclei [2] supported such an enhancement mechanism. On the other hand, in Ref. [3] the role of $E 1$ transition has been studied in the finite-temperature relativistic quasiparticle random phase approximation and it has been found that a non-negligible

a e-mail: kamila.sieja@iphc.cnrs.fr enhancement of the $E 1$ strength is present in Mo nuclei in a given temperature range. So far, calculations of both $M 1$ and $E 1$ components of radiative strength function within the same framework have not been achieved and the exact mechanism responsible for the enhancement is not well understood. Therefore, we have undertaken efforts to develop a shell model framework capable of treating both dipole components on the same footing. In this work we discuss the low lying $M 1$ strength obtained in such a shell model approach in several $p f$-shell nuclei for which exact diagonalizations for large number of states are possible. In addition to $M 1$ contribution from $p f$-shell part we calculate the $M 1$ from non-natural parity, $1 \mathrm{p}-1 \mathrm{~h}$ states built on the top of the $p f$-shell configurations. The dependence of the $M 1$ strength on the complexity of the nucleus and energy cut-off is also discussed.

\section{Theoretical framework and details of calculations}

We employ the large scale shell model framework in the model space comprising $s d-p f-g d s$ orbitals. The interaction used here is founded on a realistic set of matrix elements from a $V_{\text {lowk }}$ [4] based on the $C D$ Bonn potential [5]. The $p f$-shell matrix elements have been replaced by those of the LNPS fit [6], proven to reproduce in great detail the spectroscopy of the $p f$-shell and deformed nuclei in the island of inversion region. Further, monopole corrections have been applied to the cross-shell interactions in order to fix the known positions of single-particle/hole states (spectra of ${ }^{39} \mathrm{~K},{ }^{41} \mathrm{Ca}$ ) and the position of the lowest opposite parity-states in ${ }^{41,42} \mathrm{Ca},{ }^{44} \mathrm{Ti}$, 
${ }^{44} \mathrm{Sc}$ and ${ }^{78} \mathrm{Sr}$ nuclei. Full $p f$-shell diagonalizations for the natural parity states have been achieved and the full $1 \hbar \omega$ excitations for the opposite parity states taken into account. The latter permits exact factorization of spurious center of mass (COM) and physical (i.e., COM at rest) excitations. The shell model Hamiltonian reads:

$$
H \mathcal{D} \sum_{i} \epsilon_{i} c_{i}^{\dagger} c_{i} C \sum_{i j k l} V_{i j k l} c_{i}^{\dagger} c_{j}^{\dagger} c_{l} c_{k} C \beta H_{c . m}
$$

where the COM Hamiltonian with a multiplication coefficient $\beta$ has been added to push up the COM eigenvalues to the energy range not considered in the calculations. The diagonalization have been achieved using the Strasbourg $j$-coupled code NATHAN [7].

In the calculations of magnetic transitions the effective M1 operators have been used, i.e., the spin part of the operator has been quenched with a commonly adopted value of 0.75 [8]. This allows for a good reproduction of experimentally known magnetic moments in this region. To obtain the $B(M 1)$ averages we follow the procedure applied previously in Refs. [1,2], i.e., 60 states of each spin between 0 and 12 have been calculated for both parities. The total transition strength has been cumulated in $200 \mathrm{keV}$ bins and divided by the number of transitions in this bin to get the average $\langle B(M 1)\rangle$.

\section{Results and discussion}

We start the discussion with the calculations of the magnetic dipole averages deduced from transitions between the states in the $p f$-shell, shown in Fig. 1 as function of the transition energy $\gamma$. We compare several Ti isotopes, from $A \mathcal{D} 46$ to $A \mathcal{D} 54$, and the deformed ${ }^{48} \mathrm{Cr}$. As one can see there is a correlation between the magnitude of the low energy enhancement and the shape of the average $B(M 1)$ strength depending on the case. In the deformed ${ }^{48} \mathrm{Cr}$ there is no clear enhancement of the low lying strength. On the contrary, a rapid growth of the average strength for the lowest $\gamma$ energy is seen in the semi-magic ${ }^{50} \mathrm{Ti}$ and ${ }^{54} \mathrm{Ti} .{ }^{46} \mathrm{Ti}$ falls in between two cases with a moderate enhancement and less steep shape of the $\langle B(M 1)\rangle$. These results strengthen the conclusion from Ref. [1] that the upbend should be more probably observed in the nuclei near closed-shells with high $j$-orbits filling up, like it is the case of the neutron $f_{7 / 2}$ orbit filled in ${ }^{50} \mathrm{Ti}$, showing here the largest upbend among the studied cases. One can note that a large $M 1$ strength is located around $8 \mathrm{MeV} \gamma$ energy. This strength, corresponding to the spin-flip transitions, is also much more pronounced in semi-magic ${ }^{50,54} \mathrm{Ti}$ nuclei.

To investigate further the dependence of the upbend on the wave function composition, we have performed an additional calculation in ${ }^{46} \mathrm{Ti}$. The same number of levels was taken into account in two cases: in the first, allowing the full $p f$-shell configuration mixing, in the second, restricting to seniority 4 (the full calculation includes as well seniority 6 configurations). The result is shown in Fig. 2. The upbend is factor 2 larger when the simpler structure is imposed on the wave functions. This goes in line with the conclusions above that the complex wave functions of nucler states, like those of deformed nuclei, lead to lesser or no enhancement. One should also note from Fig. 2 that truncated shell model calculations, taking

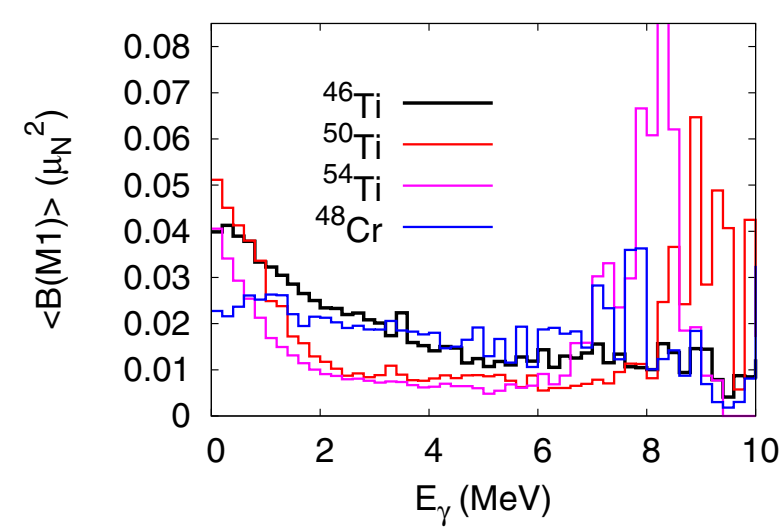

Figure 1. Averaged magnetic dipole strength in selected $p f$-shell nuclei.

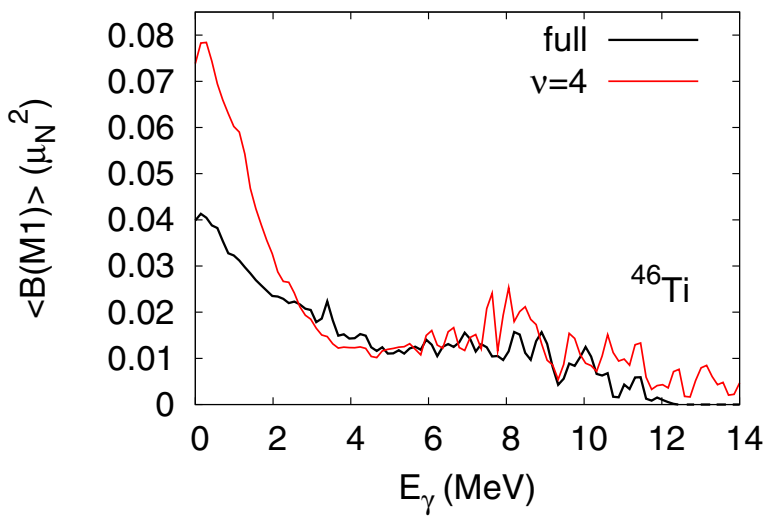

Figure 2. Averaged magnetic dipole strength in ${ }^{46} \mathrm{Ti}$. Full configuration mixing calculations in the $p f$-shell (full) is compared to the calculations truncated at seniority 4 level $(v \mathcal{D} 4)$.

into account insufficient configuration mixing or excluding necessary degrees of freedom, may exaggerate the actual magnitude of the enhancement.

In Fig. 3 we examine the dependence of the $M 1$ average strength on the cut-off in excitation energy applied in the calculations in the case of ${ }^{46} \mathrm{Ti}$. It is seen that at higher excitation energies different curves are close one to another independent of the cut-off. At lower excitation energies, in the region dominated by discrete states, there are conspicusous differences between the magnitude of strength functions. However, as far as the enhancement effect is concerned, the shape of the $\langle B(M 1)\rangle$ is always the same, pointing consistently to the upbend at low $\gamma$ energy.

Finally, we have computed also 60 states of each spin between 0 and 12 value but for the negative parity $1 \mathrm{p}-1 \mathrm{~h}$ states built on the top of the full $p f$-shell configuration mixing. In Fig. 4 we show the outcome of such a calculation in the even-even ${ }^{44} \mathrm{Ti}$ and oddodd ${ }^{44} \mathrm{Sc}$, comparing the averaged $\langle B(M 1)\rangle$ for postive parity and negative parity separately. Interestingly, the averaged strengths are very similar in shape and magnitude for both systems, irrespective the odd-odd and even-even character of the nucleus. The non-natural parity component is overally much smaller and does not upbend contrary to the natural parity one. The calculated $1 \mathrm{p}-1 \mathrm{~h}$ states are formed by lifting proton or neutron particle mostly from the $d_{3 / 2}$ orbit to the $f_{7 / 2}$ and have wave functions fragmented over many different configurations, leading to 


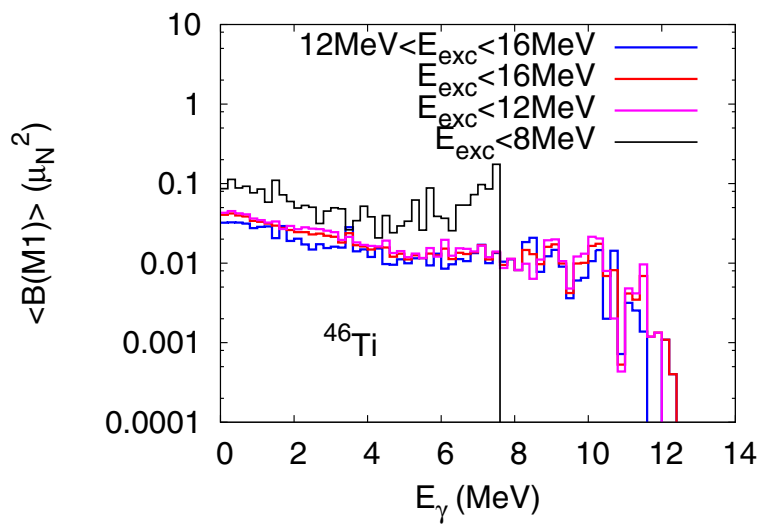

Figure 3. Dependence of the averaged $B(M 1)$ strength on the excitation energy in the case of ${ }^{46} \mathrm{Ti}$.

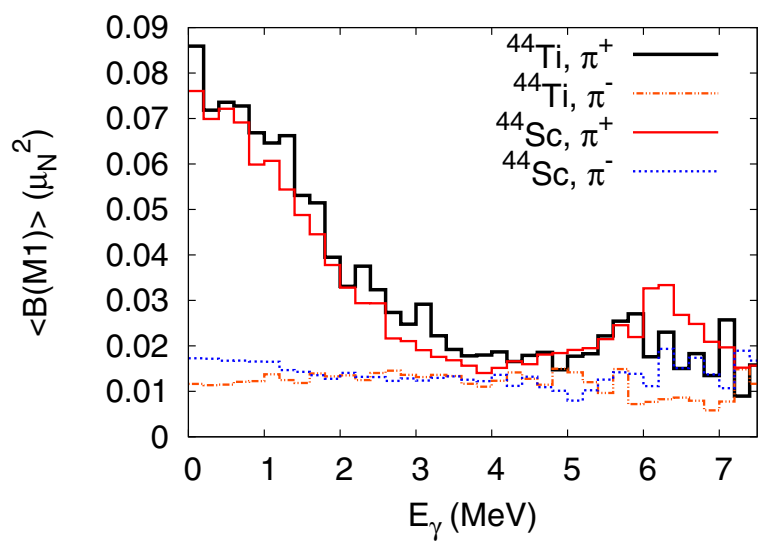

Figure 4. Averaged magnetic dipole strength in ${ }^{44} \mathrm{Ti}$ and ${ }^{44} \mathrm{Sc}$. The contributions from natural $\left(\pi^{C}\right)$ and non-natural $\left(\pi^{-}\right)$parity states are distinguished.

numerous cancellations and involving many smaller, nondiagonal matrix elements. Thus the contribution from the states of such type could moderate the magnitude of the enhancement predicted by the shell model in classical valence spaces.

In Fig. 5 we show how the present results compare to experiment in $A \mathcal{D} 44$ nuclei. Data are taken from the Oslo group [9-11]. The $\gamma$-SF has been obtained as previously in [1], i.e., $f_{M 1} \mathcal{D} 16 \pi / 9(\hbar c)^{-3}\left\langle B\left(M 1, E_{\gamma}\right)\right\rangle \rho\left(E_{i}\right)$, where $\rho_{i}$ is the level density at the energy of the initial state. The level densities and $\langle B(M 1)\rangle$ were calculated separately for both parities and the total $f_{M 1}$, plotted in the figure, was defined as the average of two components. Like for the averaged $B(M 1)$ strength, shell model predicts the same order of magnitude of the $\gamma$-SF in both systems. This leads to an excellent agreement with data at low energy for the shape and magnitude of the enhancement for ${ }^{44} \mathrm{Sc}$. At higher $\gamma$ energies the $M 1$ SF is lower than experiment, one expects however a sizeable contribution from the $E 1$ transitions as well. On the contrary, the theoretical $f_{M 1}$ in ${ }^{44} \mathrm{Ti}$ overshoots drastically experiment. This is quite a puzzling feature as the experimentally extracted $\gamma$-SF in ${ }^{44} \mathrm{Ti}$ is order of magnitude smaller than in all neighbouring nuclei, see e.g., [9-13]. The $N \mathcal{D} Z$ nucleus ${ }^{44} \mathrm{Ti}$ can exhibit features like $\alpha$-clustering, which could lower the level density and which can not be retrieved in the shell model framework. It remains however to be studied wether the origin of this large discrepancy is related solely to the problems of theoretical description.

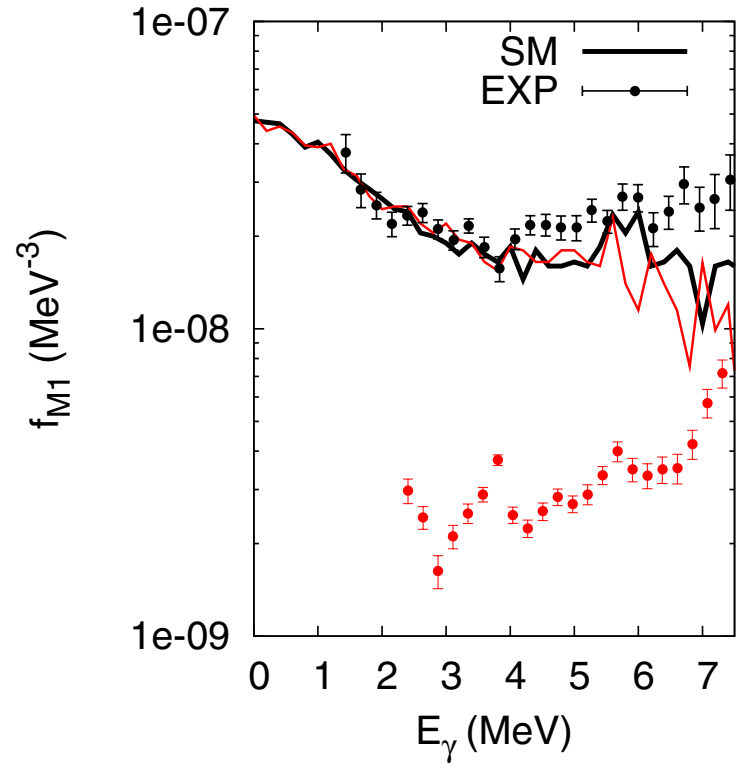

Figure 5. Radiative strength functions obtained in the present shell model compared to the Oslo data. Black points (curve) corresponds to ${ }^{44} \mathrm{Sc}$ data (calculations), the red ones to ${ }^{44} \mathrm{Ti}$.

One should note that the present shell model framework allows as well the calculations of the low lying $E 1$ dipole strength. Such calculations, currently in progress, will allow to provide fully microscopic $\gamma$ SF containing both dipole components which could be directly compared to experiment.

\section{Conclusions and perspectives}

The low energy enhancement of the dipole strength functions was studied in the nuclear shell model for selected A D 44-54 nuclei. We have presented for the first time the calculations of magnetic dipole transitions between states built of $1 \mathrm{p}-1 \mathrm{~h}$ excitations on the top of the $p f$-shell fully mixed configurations in the context of the low energy enhancement of the dipole radiation. We find that there is a considerable difference between the magnitude of enhancement and the shape of the averaged M1 strength depending on the nature of considered states, i.e. natural or non-natural parity, spherical or deformed. We have also shown that shell model calculations in too restricted model spaces can overestimate the magnitude of the low energy upbend. On the contrary, the upbend (if present) is independent on the details of calculations and the excitation energy range taken into account. The $M 1$ $\gamma$-SF obtained in our framework for ${ }^{44} \mathrm{Sc}$ agrees fairly with experiment while a large deviation is found for ${ }^{44} \mathrm{Ti}$.

Discussions and exchanges with B. Alex Brown, E. Caurier, M. Guttormsen, G. Martinez-Pinedo, A.C. Larsen, and R. Schwengner are greatefully acknowledged.

\section{References}

[1] R. Schwengner, S. Frauendorf, and A.C. Larsen, Phys. Rev. Lett. 111, 232504 (2013)

[2] B.A. Brown and A.C. Larsen, Phys. Rev. Lett. 113, 252502 (2014) 
[3] E. Litvinova and N. Belov, Phys. Rev. C 88, 031302 (2013)

[4] S. Bogner, T. Kuo, and A. Schwenk, Phys. Rep. 386, 1 (2003)

[5] R. Machleidt, Phys. Rev. C 63, 024001 (2001)

[6] S.M. Lenzi, F. Nowacki, A. Poves, and K. Sieja, Phys. Rev. C 82, 054301 (2010)

[7] E. Caurier, G. Martinez-Pinedo, F. Nowacki, A. Poves, and A.P. Zuker, Rev. Mod. Phys. 77, 427 (2005)
[8] G. Martínez-Pinedo, A.P. Zuker, A. Poves, and E. Caurier, Phys. Rev. C 55, 187 (1997)

[9] A.C. Larsen and et al., Phys. Rev. C 85, 014320 (2012)

[10] A.C. Larsen and et al., Phys. Rev. C 76, 044303 (2007)

[11] http://www .mn.uio.no/fysikk/

[12] A. Bürger and et al., Phys. Rev. C 85, 064328 (2012)

[13] N.U.H. Syed and et al., Phys. Rev. C 80, 044309 (2009) 